Stephane H Maes, (2020), "Ultimate Unification: Gravity-led Democracy vs. Uber-Symmetries", viXra:2006.0211v1, https://vixra.org/pdf/2006.0211v1.pdf or https://

shmaesphysics.wordpress.com/2020/06/16/ultimate-unification-gravity-led-democracy-vs-uber-symmetries/, June 16, 2020.

\title{
Ultimate Unification: Gravity-led Democracy vs. Uber-Symmetries
}

\author{
Stephane H. Maes ${ }^{1}$
}

June 16, 2020

\begin{abstract}
:
In this paper, we start from conventional GUTS and TOEs and discuss their challenges due not only to the lack of observations of proton decays and magnetic monopoles, but also to the fact that when gravity is considered, especially with mechanisms à la multi-folds, where gravity emerges from entanglement, both these phenomena are expected to not exist. With only a few exception, ToEs are badly hurt, including many superstrings, and most GUTS are knocked out.

Because of the massive gravity at small scale contributed by entanglement in a multi-fold universe, we encounter new lifecycles for charged black holes and discover that, at small scales, gravity is no more the weakest interaction. In fact, sources and carriers of all interactions democratically carry gravity along with their interaction the same way and all interactions have similar strength. It becomes a new symmetric state with an Ultimate Unification of all the forces, but without the hierarchies of symmetry groups typically involved in GUTs, and without intermediate GUTs not involving gravity from the get-go. These results are obtained in a multi-fold universe, but with discussions of what can be said about our real universe.
\end{abstract}

\section{Introduction}

It almost sounds like a battle of the classes and it is for the interactions... So far the tendency towards Grand Unification Theories (GUTs) and Theories of Everything (ToEs) has been to create bigger and bigger symmetry groups able to embed smaller ones. The bigger ones appear like the result of an unification of different interactions that are individually characterized by some of the smaller symmetry groups. The bigger one is assumed to have reigned at earlier times, which higher energy as well as to reign at smaller scales. Today, the separated interactions (and symmetries) are the result of symmetry breakings and phase transitions as a result. This hierarchical approach is best exemplified by the resounding success of the Electroweak unification as modeled [2] within the standard model [3].

Grand Unifications theories aim at achieving the same by expanding the process first to encompass the strong interaction [4] and then the elusive quantum gravity with Theories of Everything (ToE) [5]. Today, the latter either consists of GUTs on one side and gravity on the other, or for example with superstrings, aims at modeling everything in one shot [6]. Varieties of other ToEs exist like in [7], but they are way less popular.

However, GUTs have run into problems due to the lack of observation of proton decays [8,9], and of magnetic monopoles $[10,11]$. We claim that the addition of gravity to the standard model makes these hurdles even more critical for GUTs as well as the mainstream ToEs. By the way, [7] predicts proton decay, so it also has similar challenges.

\section{Gravity and Multi-fold Universes}

${ }^{1}$ shmaes.physics@gmail.com 
The new preprint [1] proposes contributions to several open problems in physics like the reconciliation of General Relativity with Quantum Physics, explaining the origin of gravity that proposed as emerging from quantum (EPREinstein Podolsky Rosen) entanglement between particles, detailing contributions to dark matter and dark energy and explaining other Standard Model mysteries without requiring New Physics beyond the Standard Model other than the addition of gravity to the Lagrangian. All this is achieved in a multi-fold universe that may well model our real universe, something that is still to be validated.

With the proposed model of [1], spacetime and Physics are modeled from Planck scales to quantum and macroscopic scales and semi classical approaches appear valid till very small scales. In [1], it is argued that spacetime is discrete, with a random walk-based fractal structure, fractional and noncommutative at and above Planck scales (with a 2-D behavior and Lorentz invariance preserved by random walks till the early moment of the universe). Spacetime results from random walks of particles. Spacetime locations and particles can be modeled as microscopic blackholes (Schwarzschild for photons and spacetime coordinates, and between Reisner Nordstrom [12] and Kerr Newman [13] for massive and possibly charged particles - the latter being possibly extremal). Although surprising, [1] recovers results consistent with other like [25], while also being able to justify the initial assumptions of black holes from the gravity or entanglement model. The resulting gravity model recovers General Relativity at larger scale, as a 4-D process, with massless gravity, but also massive gravity components at very small scale that makes gravity significant at these scales. Semi-classical models also work well till way smaller scales that usually expected.

It means that, following [1], gravity can be added to the Standard Model Lagrangian [14] by adding gravity and entanglement terms.

\section{Decimation of GUTs, ToEs, Including Many Superstring Theories}

$[1]$, and derived papers $[9,10]$, show that, with gravity, there are little chances of ever encountering proton decay or a magnetic monopole, as elementary particles. So, it is not just an issue of waiting for an observation, but a motivated reasoning that we may be waiting in vain, and meanwhile, spending lots of resources on the search.

Worse, as discussed in [1,15], ToEs that model gravity are under tremendous stress: they are essentially obtaining the wrong results as, so far, if they predict proton decays and magnetic monopoles, despite gravity rendering these phenomena improbable. The theories affected include many superstrings (and therefore possibly M-theory) but not all $[22,23]$. Some suggestions to address these challenges are discussed in [15].

GUTs are not faring much better: they all fail on the magnetic monopole and only a few approaches, so far not widely popular, avoid predicting proton decays $[23,24]$. Worse, their actual proton half-time predictions are being blown off one by one by the lack of observation of proton decays [21]. Some of the thoughts about supersymmetry [15], and related considerations for GUTs [11] may provide ways forward.

In any cases, it seems like the wind has changed against hierarchies of bigger and bigger symmetry groups swallowing smaller one and reigning for a while (in time or in a scale range). The electroweak unification was where that strategy played out succesfully. Trying it again may not be the right approach.

Gravity is not just unifying "later", as envisaged by these models, but it is also preventing many of these symmetry schemes beyond the Electroweak unification. In fact, we could try an intuitive reasoning that shows that, maybe, it could have been guessed all along. Indeed, the electroweak scheme along with its Higgs mechanism gives mass to all the Standard Model elementary constituents, starting from massless particles (fermions and bosons) before the Electroweak symmetry breaking with the Englert-Brout-Higgs-Guralnik-Hagen-Kibble mechanism [16]. Therefore, physical interpretations seem to immediately throw a red flag that, so far, we haven't seen really considered anywhere in the literature. While possible of course, it seems that repeating the same scheme earlier (earlier in time or at smaller scales) with whatever variations on the Higgs, leads to an attempt at recurrent mass generation. It simply does not make too much sense, and it looks like nature agrees with us. Indeed, in the example of the 
Georgi- Glashow model, there are inconsistencies in the Higgs elements that do no match reality, as we just anticipated by our thought considerations [17]. Similarly the scheme in $\mathrm{SO}(10)$ seems unstable in terms of its Higgs [18]. We may be onto something with our reasoning, ... We haven't checked the other schemes, but we are ready to guess that the same kind of issues occur. All these considerations are in addition to the proton decay and magnetic monopole issues.

Note that the last paragraph reflects an intuitive thought process. It is not the result of a mature investigation. Yet, in our view, it physically explains why GUTs, while esthetically pleasant as elaborate mathematical formulations, might not have a clear place in Physics; at least in a multi-fold universe.

\section{Black Holes in a Multi-fold Universe and Some Weak Gravity}

\section{Digression}

If we go back to [1], it analyzes the reasoning behind the Weak Gravity Conjecture (WGC) [19]. Indeed, [1] discovers additional small-scale massive gravity contributions at very small scales (as well as entanglement contributions). They result from entanglement of massive virtual particles pairs around sources of energy (particles). It adds evolving stronger and stronger contribution towards smaller scales that match the range of these particles and their interactions. As a result, at small scales, gravity has a larger impact, not just because distances are short but because of these additional massive contributions. Therefore, it is reasonable to wonder if the conventional WGC is still correct at very small scales.

Reverting to the original WGC proof in [19], [1] notices that if massive gravity is added, it amounts to increasing the gravitational constant $\mathrm{G}$ (in first approximation) and therefore reducing entropy for black holes, in a way that it increases when small black holes are smaller. A different trend for extremal blackholes from what is predicted, for small blackholes, with conventional gravity considerations as in [19].

One also needs to remember that [1] models particles (and spacetime locations) with microscopic black holes (for its reconstruction phase). Doing so, charged elementary particles like electrons appear like an extremal microscopic black hole.

The conclusion is that extremal black holes can disintegrate into pairs of extremal black holes etc. Therefore, according to [1], a new lifecycle is proposed for black holes (when they get charged ): they eat matter and evaporate till they become (charged then) extremal. Then, instead of the conventional evolution (proposed in [19]) where they split into an extremal black hole and a smaller non extremal black hole, [1] prescribes that they will disintegrate into smaller extremal black holes till reaching the smallest suitable charged elementary particle. Doing so we have no remnant trouble and no paradox. The remnants are the elementary (stable) particles.

\section{Ultimate Unification}

Doing so shows that instead of (strict) inequalities of charges per mass encountered in [19]. In [1], we converge towards an equality $\mathrm{m}=\mathrm{q}$ (all the relevant charges) in extremal black holes. Following the same kind of handwaving as [19], it means that, at very small scales or in extremal conditions, the strength of gravity matches all the other interactions. Physically and intuitively (based on the model if [1], where gravity is carried by pairs of entangled particles), it means that each particle, carrying its own charges and/or interactions equally also carries gravity effects and so contribute equivalently to whatever it interacts with and for gravity: we have democracy of the effects and all the interactions converging. By the way, it makes sense as above the electroweak (without a GUT; above the GUT scales otherwise), everything becomes massless (to be understood as long range when energy are high enough or behaving as if all interactions are long range when looking at very small scales otherwise). This effect is progressively starting below the electroweak scales. 
The reasoning works for combinations of the strong and electroweak interactions or individually. So all interactions have essentially the same intensity. It reminds of the GUT analogous picture, showing the convergence of their coupling constants at larger energies, that was used to justify GUTs and what are the expected GUT scales (e.g. see convergence of the couplings in $[19,20])$. But now, it is for all interactions and there is probably no GUT intermediary step, per our considerations earlier.

It is what [1] calls the Ultimate Unification (UU). It is not the result of an all-encompassing symmetry that has swallowed a GUT symmetry. It is rather that all interactions contribute equivalently to their effects and gravity, which is another symmetry.

The WGC is not satisfied at very small scales in a multi-fold universe. Instead, gravity becomes of the same order as the other interactions, all converging to a same intensity.

\section{Conclusions}

[1] and the present paper, show that a Ultimate Unification remains possible within a multi-fold universe. It is the result of a democratization of the roles to carry gravity along with other interactions, and not an uber-symmetry scheme. A new symmetry exists: all interactions have the same intensity with same contribution from all particles as sources and carriers. Things have simplified a lot which is also what was expected for Planck Scale Physics. All particles are massless, so at high energy, the behavior is also at all ranges. At very small scales, it happens within the range where UU plays as if their range were infinite. We have democratization of entanglement and gravity effects across all sources and carriers.

Superstrings are not done in our view and neither is LQG and other constructive gravity models. But they may benefit from the lessons learned from [1]. See [15] for an overview analysis for superstrings. LQG will be addressed in an upcoming paper.

As discussed above, in multi-fold universes, gravity got its revenge against GUTs (e.g. for leaving it out of the GUT intermediary step) and shredded its label of always being the weakest interaction. GUTs have no clear places anymore because of gravity: it mangles the symmetries needed for monopoles, it flips the chirality to smear symmetry anomalies and doing so reestablishes anomalous symmetries that prevent proton decays, it gets its charge (i.e. the mass) from the Higgs mechanism and principle of equivalence (also explored in [1]) and throws a fit for not being invited to the GUT party, by not being able to cleanly support recurrent mass generations (per the discussion above, maybe we get only one chance at generating elementary masses - note this is not the same as the mass generation of quark composite, which is not what we discuss here). These latter considerations force different unification schemes than uber-symmetries (Especially if GUT symmetry breaking was needed in the big bang chronology e.g. to explain the onset of inflation - we need another phase transition story; it will be discussed in an upcoming paper), where gravity get equal treatment with everybody else equally contributing to its effects.

We believe that the UU outcome is a remarkable result. Frankly, it was totally unexpected after [1] seemed to dis the prospects of many ToEs and GUTs. While developing [1], it felt as if the big bang could no more show something worth mentioning related to Unification above the electroweak symmetry breaking, other than an handwaved and unexplained gravity decoupling. For us, GUTs and ToEs were not even envisaged in a multi-fold universe. Looking at WGC changed everything. Now, it has all been clarified. And the unification goal can been achieved with UU, at least within multi-fold universes. After all, it was Einstein's long dream and it is the Holy Grail of Physics. We just still have to make sure that our universe can be modeled as a multi-fold universe.

If it can't be shown that our real universe is well modeled by multi-folds mechanisms, or if it isn't, much of the reasoning presented here may still remain valid when gravity is strong enough at sufficiently small scales. Isn't it what everybody expects for gravity around Planck scale? What about at a big higher scales, but below the ranges of the electroweak and strong interactions? If it is strong enough, then our analysis is probably repeatable for our 
real universe. So, no matter what, no break for all the challenges we have thrown at all these other theories out there: the arguments presented here should be evaluated against each theory and addressed, no matter what happens with our multi-fold theory. The very recent developments that no extra dimensions would exist based on gravitational wave analyses, is certainly also not good news at all for superstring theories [26]. The bad news keep on coming. Hopefully [15] can help a bit to bring back some relief and comfort, as it provides a positioning for superstrings with respect to our spacetime, albeit it is probably a far cry from ambition for superstrings.

References: (most references come from popular science to make the discussion more approachable. See [1] for more references.)

[1]: Stephane H. Maes, (2020) “Quantum Gravity Emergence from Entanglement in a Multi-Fold

Universe", viXra:2006.0088v1, (June 9, 2020).

[2]: https://en.wikipedia.org/wiki/Electroweak interaction

[3]: https://en.wikipedia.org/wiki/Standard Model

[4]: https://en.wikipedia.org/wiki/Grand Unified Theory

[5]: https://en.wikipedia.org/wiki/Theory of everything

[6]: https://en.wikipedia.org/wiki/Superstring theory

[7]: https://en.wikipedia.org/wiki/An_Exceptionally_Simple_Theory_of_Everything

[8]: https://en.wikipedia.org/wiki/Proton decay

[9]: Stephane H Maes, (2020), "Gravity Induced Anomalies Smearing in Standard Model so that Protons May Never

Decay, Except in Black Holes", viXra:2006.0128v1, https://shmaesphysics.wordpress.com/2020/06/12/protonsmay-never-decay-except-in-black-holes/, June 13, 2020.

[10]: https://en.wikipedia.org/wiki/Magnetic monopole

[11]: Stephane H Maes, (2020), "Gravity or Magnetic Monopoles? You Cannot Have Both!", viXra:2006.0190v1, https://shmaesphysics.wordpress.com/2020/06/15/gravity-or-magnetic-monopoles-you-cannot-have-both/, June 15, 2020.

[12]: https://en.wikipedia.org/wiki/Reissner\%E2\%80\%93Nordstr\%C3\%B6m metric

[13]: https://en.wikipedia.org/wiki/Kerr-Newman metric

[14]: https://en.wikipedia.org/wiki/Mathematical formulation of the Standard Model

[15]: Stephane H Maes, (2020), "Dualities or Analogies between Superstrings and Multi-fold Universes", viXra: 2006.0178v1, https://shmaesphysics.wordpress.com/2020/06/14/dualities-or-analogies-between-superstringsand-multi-fold-universes/, June 14, 2020.

[16]: https://en.wikipedia.org/wiki/Higgs mechanism

[17]: https://en.wikipedia.org/wiki/Georgi\%E2\%80\%93Glashow model

[18]: https://en.wikipedia.org/wiki/SO(10) (physics)

[19]: Nima Arkani-Hamed, Lubos Motl, Alberto Nicolis, Cumrun Vafa, (2006), "The String Landscape, Black Holes and Gravity as the Weakest Force", arXiv:hep-th/0601001v2.

[20]: Sabine Hossenfelder, (2018), "Lost in Math: How Beauty Leads Physics Astray", Basic Books.

[21]: https://www.quantamagazine.org/no-proton-decay-means-grand-unification-must-wait-20161215/

[22]: Jogesh C. Pati, (1996), "Baryon Non-Conservation in Unified Theories, in the Light of Supersymmetry and Superstrings", arXiv:hep-ph/9611371v1

[23]: https://ncatlab.org/nlab/show/proton+decay

[24]: G.Lazarides, C.Panagiotakopoulos, Q.Shafi, (1993), ), "Supersymmetric Unification without Proton Decay", arXiv:hep-ph/9306332v1

[25]: Burinskii, Alexander, (2008), "The Dirac-Kerr-Newman electron", arXiv:0507109v4

[26]: Kris Pardo, Maya Fishbach, Daniel E. Holz, David N. Spergel, (2020), "Limits on the number of spacetime

dimensions from GW170817", arXiv:1801.08160v3 